\title{
Inclusão, um cromossomo à mais (Síndrome de Down ) na educação
}

\author{
Inclusion, one more chromosome (Down Syndrome) in education \\ Inclusión, un cromosoma más (Síndrome de Down) en la educación
}

Recebido: 02/11/2021 | Revisado: 09/11/2021 | Aceito: 02/12/2021 | Publicado: 13/12/2021

\author{
Karla Pinheiro de Moraes \\ ORCID ID: https://orcid.org/0000-0001-9843-4947 \\ Faveni Faculdade Venda Nova do Imigrante, Brasil \\ E-mail: karlapinheiromoraes@gmail.com
}

\begin{abstract}
Resumo
O presente artigo tem como objetivo de investigar a Inclusão do aluno com um cromossomo a mais no caso a chamada Síndrome de Down na educação. Atualmente se fala muito sobre a Educação Inclusiva, e dentro desta modalidade escolar, um dos assuntos que se tem interesse de se compreender são os conceitos da Síndrome de Down. A SD não é uma doença, mas sim um estado uma condição irregular á pessoa, uma alteração genética, que impossibilita o desenvolviento normal como os outros individuos saudáveis. Porém, envolve questões de saúde, desde o nascimento a criança e deve ser acompanhada por profissionais qualificados. Cada pessoa reage no seu tempo de uma forma diferenciada ao tratamento, conforme sua genética, sua forma de interagir, ocorrerá sua evolução de acordo com suas necessidades. Pode-se dizer que as pessoas com SD são mais parecidas do que diferentes. Sem sombra de dúvidas a escola é considerado um ambiente ideal para que se possa construir e transmitir conhecimentos como a cultura e moldar cidadãos, porém, para receber uma criança SD, porém é indispensável que a escola tenha profissionais maior qualificados e materiais pedagógicos para a educação especial. Com isso, a finalidade maior desta pesquisa é o desenvolvimento dos alunos com um cromossomo a mais na vida escolar. Contamos com a nossa Constituição Federal que assegura que todos os cidadãos, sem exceção, de crenças, cor, raça, tem direto à educação, inclusive as pessoas com deficiências, no caso a Síndrome Down, observa-se que mesmo que incluídos no ensino regulas, bem recebidos, não se pode dizer que suas necessidade estão satisfatórias, pois, pode ocorrer a falta de estrutura física das escolas, como: acessibilidade e professoress despreparados. Como também, a falta de uma equipe, multidisciplinar que conta com: Psicólogos, Psiquiatras, Terapeutas Ocupacionais, Fisioterapeutas, Psicopedagogos e Fonoaudiólogos, que devem realizar o acompanhamento dos alunos com SD. É muito importante esse trabalho para que as pessoas com SD dever ser inclusas na sociedade, não por por uma lei, mas sim por serem humanos, e dentro de suas condições compartilham, carinho, amor, aprendem e interagem com pessoas chamadas de "normais". Vale ressaltar da necesscidade de alertar as famílias, os educadores e a sociedade, das características dos portadores da SD, demonstrando como eles podem lidar e acabar com preconceitos e conscinetizar que eles são capazaes e produtivos.
\end{abstract}

Palavras-chave: Inclusão; Educação; Síndrome de Down; Preconceito; Profissionalismo e acessibilidades.

\begin{abstract}
This article aims to investigate the inclusion of the student with an extra chromosome in this case the so-called Down Syndrome in education. Currently, there is a lot of talk about Inclusive Education, and within this school modality, one of the subjects that one is interested in understanding is the concepts of Down Syndrome. DS is not a disease, but a condition, an irregular condition to the person, a genetic alteration, which makes normal development impossible like other healthy individuals. However, it involves health issues, from birth to the child and must be accompanied by qualified professionals. Each person reacts in their own time in a different way to the treatment, according to their genetics, their way of interacting, their evolution will occur according to their needs. It can be said that people with DS are more alike than they are different. Without a doubt, the school is considered an ideal environment for building and transmitting knowledge such as culture and shaping citizens, however, to receive a SD child, it is essential that the school has more qualified professionals and teaching materials for education Special. Thus, the main purpose of this research is the development of students with an extra chromosome in school life. We have our Federal Constitution which ensures that all citizens, without exception, of beliefs, color, race, have the right to education, including people with disabilities, in this case Down Syndrome, it is observed that even if included in regular education, well received, it cannot be said that their needs are satisfactory, as there may be a lack of physical structure in schools, such as: accessibility and unprepared teachers. As well as the lack of a multidisciplinary team that counts with: Psychologists, Psychiatrists, Occupational Therapists, Physiotherapists, Psychopedagogists and Speech-Language Pathologists, who must monitor the students with DS. This work is very important so that people with DS should be included in society, not because of a law, but because they are human, and within their conditions they share, affection, love, learn and interact with people called "normal". It is worth emphasizing the need to alert families, educators and society about
\end{abstract}


the characteristics of DS patients, demonstrating how they can deal with and eliminate prejudice and make them aware that they are capable and productive.

Keywords: Inclusion; Education; Down's syndrome; Prejudice; Professionalism and accessibility.

\section{Resumen}

Este artículo tiene como objetivo investigar la inclusión del alumno con un cromosoma extra en este caso el llamado Síndrome de Down en la educación. Actualmente se habla mucho de Educación Integrada, y dentro de esta modalidad escolar, una de las asignaturas que a uno le interesa comprender son los conceptos de Síndrome de Down. El SD no es una enfermedad, sino una condición, una condición irregular para la persona, una alteración genética, que imposibilita el desarrollo normal como otros individuos sanos. Sin embargo, involucra cuestiones de salud, desde el nacimiento hasta el niño y debe ir acompañado de profesionales calificados. Cada persona reacciona en su propio tiempo de forma diferente al tratamiento, según su genética, su forma de interactuar, su evolución se producirá según sus necesidades. Se puede decir que las personas con SD son más parecidas que diferentes. Sin duda, la escuela es considerada un entorno ideal para construir y transmitir conocimientos como la cultura y formar ciudadanos, sin embargo, para recibir a un niño SD, es fundamental que la escuela cuente con más profesionales calificados y materiales didácticos para la educación Especial. Así, el objetivo principal de esta investigación es el desarrollo de estudiantes con un cromosoma extra en la vida escolar. Contamos con nuestra Constitución Federal que asegura que todos los ciudadanos, sin excepción, de creencias, color, raza, tienen derecho a la educación, incluidas las personas con discapacidad, en este caso el Síndrome de Down, se observa que incluso si se incluye en la educación regular, bien recibido, no se puede decir que sus necesidades sean satisfactorias, ya que puede haber una falta de estructura física en las escuelas, tales como: accesibilidad y docentes no preparados. Así como la falta de un equipo multidisciplinario que cuente con: Psicólogos, Psiquiatras, Terapeutas Ocupacionales, Fisioterapeutas, Psicopedagogos y Fonoaudiólogos, quienes deben monitorear a los estudiantes con SD. Este trabajo es muy importante para que las personas con SD sean incluidas en la sociedad, no por una ley, sino porque son humanos, y dentro de sus condiciones comparten, afecto, aman, aprenden e interactúan con personas llamadas "normales". Cabe destacar la necesidad de alertar a las familias, educadores y sociedad sobre las características de los pacientes con SD, demostrando cómo pueden afrontar y eliminar los prejuicios y concienciarlos de que son capaces y productivos.

Palabras clave: Inclusión; Educación; Sindrome de Down; Perjudicar; Profesionalismo y accesibilidad.

\section{Introdução}

Este trabalho tem como objetivo principal investigar a inclusão do aluno com um cromossomo a mais, no caso a chamada Síndrome de Down na educação regular. No Brasil a inclusão escolar teve seu início na década de 90, século passado, iniciando com o princípio do direito à diferença, tendo a perspectiva de que todas as crianças devem aprender juntas. Com a nova construção do sistema educacional inclusivo, foi impulsionadado com alguns apontamentos legais como: a Constituição Brasileira de 1988, a Declaração Mundial sobre Educação para Todos e a Declaração Mundial de Salamanca (Brasil, 1997; Unesco, 1990).

Neste artigo, trataremos de um tipo de aluno que necessida dessa estrutura o aluno portador da Síndrome de Down (SD), pois, para sua inclusão na escola são necesssárias ações complexas e adaptações físicas e curriculares, em relação a qualificação dos professores. A Síndrome de Down (SD) é causada pela presença de três cromossomos 21 em todas ou na maior parte das células de um indivíduo. Ocorrendo na concepção da criança.

As pessoas com SD, ou trissomia do cromossomo 21, têm 47 cromossomos em suas células em vez de 46, parecida com a maioria da população. Porém, as crianças, os jovens e os adultos com SD podem ter características semelhantes e ficarem sujeitos a maiores incidências de doenças, contudo elas apresentam personalidades e características diferentes e únicas peculiares a sua necessidade. Dessa forma, para incluir crianças com SD na Educação escolar requer, estruturas necessárias, e professores específicos qualificados. (Voivodic, 2008).

De acordo com a Política Nacional de Educação Especial, apresentada em 2008, se tratando de educação inclusiva, busca integrar a proposta pedagógica da escola regular a inclusão escolar de alunos com deficiência, com transtornos globais do desenvolvimento e altas habilidades na educação regular de ensino, garantindo direito à aprendizagem, participação e continuação nos níveis mais elevados de ensino (Brasil, 2008). 
Pode-se dizer que a Política Nacional de Educação, auxiliou bastante no sistema educacional brasileiro, houve novos avanços na educação escolar de crianças com Síndrome de Down. Porém, nota-se vários desafios como: uma educação adequada aos portadores de deficiência, que toprn possível o acesso, ingresso e a permanência desses alunos e educandos especiais mediante a educação inclusiva e ideal, com uma educação de qualidade para todos sem distinção de raça, cor ou credo, e a realidade das condições atuais dos sistemas escolares.

No decorrer do trabalho, trataremos de um dos tipos de aluno atendido nessa proposta é o aluno com SD. A SD é um acidente genético que ocorre na alteração do par do cromossomo 21, quando surge um cromossomo extra. A pessoa com SD apresenta diversas características como: atraso no seu desenvolvimento funcional, físico e intelectual, dificuldade de socialização, entre outros fatores, que nos leva a investigar a inclusão na educação destas pessoas, que tem um crisma incível e são bem inteligetnes e produtivos, com este trabalho será possível, que outros estudantes, tenha uma fonte de informação para realizar outras pesquisas, relevantes a este tema.

\section{Metodologia}

Tratou-se de uma pesquisa de revisão integrativa de literatura, onde objetiva-se abordar aspectos relevantes através de pesquisa com opiniões de diversos autores que conhecem sobre o tema, na tentativa de identificar e favorecer a ampliação de estudos e conhecimentos a respeito do tema abordado uma pesquisa qualitativa, com os pontos positivos e os negativos dos autores. (Santos Filho, 2001).

Foram avaliadas publicações científicas indexadas nas bases de dados Scielo, Google Acadêmico, LILACS e Base de Dados em Enfermagem, acessadas por meio da Biblioteca Virtual em Saúde, Teses sobre o tema, livros e Artigos de Universidades Nacionais, em março de 2020. Seguindo os critérios de inclusão, selecionaram-se sete estudos com publicações entre 2008 e 2020. Os dados foram submetidos à análise de conteúdo.

Para a realização da pesquisa foram utilizados métodos de pesquisa científica embasados em autores que tratam do tema. Para tanto, quanto aos meios, a pesquisa constitui-se de base bibliográfica. A pesquisa Bibliográfica evidencia-se a cobertura, pelo pesquisador, de uma ampla ambrangência de fenômenos principalmente quando o problema da pesquisa aborda dados muito dispersos no universo pesquisado.

\section{Compreensão, Características e Necessidades: Amparadas pela LDB (Lei Federal nº 9.394/96).}

Para iniciar nosso assunto, fala-se da Lei de Diretrizes e Bases da Educação Nacional, LDB, N. 9394/96, que descreve no artigo 58 , o conceito de educação especial, no inciso $1^{\circ}$ e serviços e no inciso $2^{\circ} \mathrm{o}$ atendimento: Art. $58^{\circ}$. Onde se entende o porquê da educação especial, a modalidade de educação escolar, oferecida preferencialmente na rede regular de ensino, para educandos portadores de necessidades especiais. $\S 1^{\circ}$. Haverá, quando necessário, serviços de apoio especializado, na escola regular, para atender às necessidades da clientela de educação especial. § $2^{\circ}$. Na Constituição Federal de 1988 (CF/88), que em seu artigo 208, parágrafo III diz: que o dever do Estado com a educação é efetivado através de atendimento especializado. (Brasil, 2008).

Desta forma, fica clara a importância da educação como fator de transformação para todos os indivíduos, com excelência e qualidade, ou seja, que supra às necessidades educativas especiais, incluído os indivíduos com deficiência mental. Neste caso, "as crianças com Síndrome de Down têm a deficiência mental como uma de suas características mais constantes e, portanto, é evidente a complexidade e a importância de sua educação" (Voivodic, 2013, p.17).

Ainda segundo Voivodic (2013). Afirma que as crianças com Síndrome de Down levam mais tempo para se desenvolver, dessa forma necessitam de um currículo mais detalhado, com propostas pedagógicas que facilitem seu aprendizado. Geralmente estas crianças possuem, de forma generalizada, uma aprendizagem com pontos fortes e fracos. 
Porém, está atualizado com estas características, com certeza facilita o aprendizado desse público, permitindo aos e aos docentes planejar e aplicar com atividades importantes, significativas e programas de trabalho.

Afirma-se que, a escola deve ofertar oportunidades para que as crianças com SD possam desenvolver as suas habilidades sociais e assim criarem uma sensação de identidade pessoal, auto estima, respeito e prazer. Para que isso ocorra é indispensável um relacionamento com os pais e familiares dos alunos, fazendo com que haja interação e assim, e eles repassem a rotina e necessidade dos alunos da escola, facilitando o processo de aprendizagem e até nas tomadas de decisão, para planejamento, gerando benefícios não só para as escolas, mas para todos os envolvidos neste processo. (Pueschel,1993).

De acordo com as leis que beneficiam a inclusão das crianças com deficiência, incluindo as com o SD, fica nítido, que é indispensável, que todas as crianças tem o direito de estudar, como também, para que as escolas recebam estas crianças, precisam estar preparadas para este acolhimento, com uma estrutura física e profissional adequada, para a acessibilidade das mesmas, e uma equipe multidisciplinar de profissionais capacitados, para lhe dar com as diferenças pedagógicas destes alunos, tão especiais.

\subsection{Pontos cruciais sobre a inclusão escolar}

Pode-se dizer que falar de inclusão atualmente na sociedade é um desafio. Isso ocorre, pelo fato desta sociedade definir regras para que estes alunos especiais estudem juntamente com os alunos ditos "normais". Além disso, infelizmente em pleno século ainda existe o chamado preconceito, lamentável, e também a falta de verbas públicas para que seja investido em adequaçoes estruturas físicas e na qualificação dos professores para melhor atender estas crianças especiais que possui tantos direitos que devem ser respeitados por toda a sociedade.

Através de pesquisas de diversos autores, pode-se afirmar que a inclusão de alunos com deficiência na educação, é considerado um dos problemas mais discutidos no âmbito da educação brasileira. A educação inclusiva está relacionada à uma conquista de um ensino de excelência para todos os alunos, ao buscarem acesso e permanência contínua, podendo encontrar as escolas com a estrutura adequada para terem uma rotina escolar. Como também, profissionais qualifcados para trabalhar estas diferenças. (Ferraz et al., 2010; Mendes, 2006; Sousa, 2007).

Segundo Fernandes (2006, p. 45) afirma que a inclusão "é um movimento ligado à valorização de pessoas, independentemente de suas diferenças individuais, inclusive àquelas com deficiências". Como também, promover a inclusão social é integrar as pessoas dentro da sociedade, dessa forma proporcionar a igualdade de oportunidades no que diz respeito à educação, isto é, para que a inclusão social das pessoas com necessidades especiais ocorra de fato e de direito, são necessárias atuações que combatam eficazmente o preconceito e a desigualdade existentes a sociedade e, acredita-se que através da escola se atinge o melhor caminho para a inclusão. Na mesma direção, Stainback e Stainback (1999, p. 21) apud Martins (2011, p. 19), afirma que.

...a educação inclusiva pode ser definida como "a prática da inclusão de todos independentes de seu talento, deficiência, origem socioeconômica ou cultural- em escolas e salas de aulas provedoras, onde as necessidades desses alunos sejam satisfeitas". Para tanto, essas pessoas são amparadas por lei "o atendimento aos alunos com necessidades especiais deve ser realizado em classes comuns do ensino regular, em qualquer etapa ou modalidade a educação básica" (Brasil apud Gões e Laplane, 2004, p. 23).

Assim, pode-se dizer que as dificuldades existem, neste processo educativo, porém, com esforço necessitam ser superadas, pois isso não deve impedir que a inclusão ocorra, concordamos Gões e Laplane (2004) ao afirmarem que a inclusão já prevista em lei e na constituição, deve ser executada como um direito, sendo considerado um crime quando por algum motivo, as escolas não podem receber estes alunos com necessidades especiais. 
Conforme os autores, pode se dizer que o processo da Inclusão social, já vem tomando espaço na atualidade, processo este que busca incluir as pessoas com necessidades especiais no ensino regular de educação e em nossa sociedade em geral. Porém, observa-se uma série de dificuldades encontradas pelo caminho, como: Falta de estruturas físicas adequadas, falta de qualificação dos professores, itens que, são essências para que este processo de certo e as pessoas com deficiência, incluindo as com SD, possam estudar no ensino regular de forma segura e contínua.

\section{O Aluno com Síndrome de Down (SD) e a Inclusão Escolar}

Estudantes com SD têm mais possibilidades de desenvolvimento quando percebidos como pessoas capazes de receberem estimulação pedagógica adequada; para isso, é necessário que o sistema de ensino considere-o detentor de potenciais de aprendizagens, de características próprias e comportamentos diferenciados que devem ser superestimados e aprimorados pelo próprio processo educacional. Para tanto as características da escola deve ser conpatível e o tipo de ensino específico deve ser disponibilizado para que possa se intensificar as dificuldades de aprendizagem (Silva, 2010).

A SD ou trissomia 21 é uma condição genética, um desequilíbrio na formação cromossômica, com mais um cromossomo no par 21. As pessoas com SD têm 47 cromossomos, em vez de 46. As características típicas destas pessaos são: desenvolvimento motor e cognitivo mais lento, deficiência intelectual, rosto com perfil achatado, pálpebras com inclinação para cima, orelhas pequenas, arredondadas e displásicas, alterações na linguagem, motricidade e interação. Estas características, se manifestam de maneira distintas entre os sujeitos, e seu aspecto psicossocial deve ser avaliado, individualmente e não tem um tempo fixo para a construção da aprendizagem. A construção das habilidades e a superação das limitações dependem de um ambiente estimulante e favorável (Castro; Pimentel, 2009;).

Pode-se dizer que o contexto familiar é de suma importância para o desenvolvimento e para ajudar na educação da pessoa com SD, uma vez que a interação é muito positiva entre pais e filhos promove efeitos relevantes para o progresso das áreas cognitivas, linguísticas e socioemocionais da criança com SD. Pois, com a interação dos adultos e colegas mais desenvolvidos, a criança recebe instrumentos e estratégias psicológicas aprimoradas por meio da internalização que vai proporcionar aprendizagens significativas. (Voivodic, 2005).

De acordo com os autores acima a SD não deve se constituir num entrave se houver oportunidades educacionais. É pode se observar que é de grande importância a superação das dificuldades devido as deficiências, levando em consideração seus ritmos de aprendizagem e respeitadas as caraterísticas peculiares, de cada um, pois se manifestam individualmente.

\subsection{A escola e inclusão}

Ao falar-se de Inclusão, se trata de Educação Especial que é destinada aos portadores de deficiência como: visual e auditiva, motora, cognitiva, doenças crônicas, autistas, Síndrome de Down, inadaptação social entre outras, pois precisam de procedimentos metodológicos e recursos especiais para sua permanência na escolar regular. Pode-se dizer que no decorrer da história foram tratados como doentes. Não havia respeito, ao contrário, e eram tratadas com desrespeito, abandono independente da deficiência, física ou mental.

Segundo Aranha, (2001, p.2). A educação evolui a cada dia e tem papel fundamental, por favorecer todos os cidadãos que tem acesso ao conhecimento e desenvolvimento. Porquê é na escola que se forma cidadãos que através dos conteúdos curriculares, adquirem a formação crítica e cognitiva. No entanto a escola necessita se adequar e oferecer boa qualidade de ensino que contribua no processo de desenvolvimento de todos os alunos, a partir da educação básica até o ensino superior.

Diante disto, a escola deve e tem que estar preparada ser vista como espaço para todos, visando sempre soluções para o acesso de todos os alunos, crianças e adolescentes. "A ideia de inclusão se fundamenta numa filosofia que reconhece e aceita a diversidade na vida em sociedade". (Aranha, 2001, p.2). 
Figura 1 - Inclusão escolar.

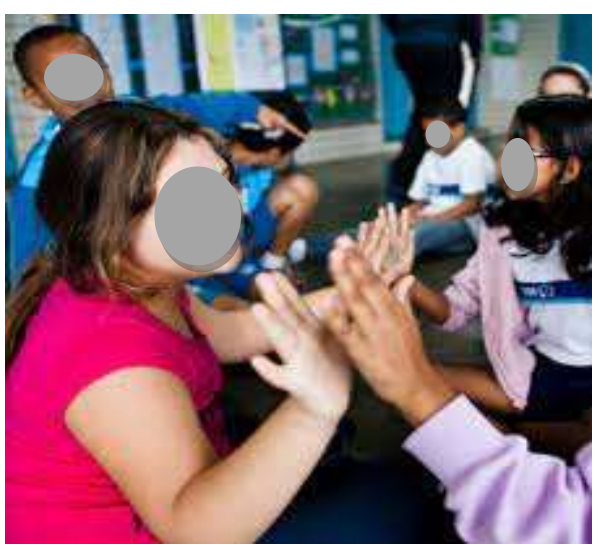

Fonte: http://cerbrum.com/inclusao-escolar-promove-justica/

Para Mantoan, (2003, p 22). A inclusão escolar refere-se em aprimorar e qualificar o ensino das escolas de ensino regular, para beneficiar todos os alunos com necessidade especiais. "A Constituição, garante a educação para todos e isso significa, para todos mesmos e, para atingir o pleno desenvolvimento humano e a cidadania, entende-se que essa educação não pode se realizar em ambientes segregados".

De acordo com a autora, A educação é para todos, independente de raça, cor, idade, ou gênero, tipo de necessidades especiais, que a oportunidade de educação deve ser igualitária. Assim, pode-se afirmar que a educação inclusiva é um grande desafio para todos, sociedade e escola. O que se espera na verdade é uma boa educação com garantias ao aluno dito "normal" e os especiais um conhecimento que possibilite sua inserção na sociedade.

Como pode-se se observar, com os relatos dos autores ao se falar de Inclusão social, se trata de reconhecer que são necessárias as mudanças sociais, que elas estão evoluindo a cada dia, para que se possa lidar com as diversidade e diferenças, é um grande desafio para a escola como para os educadores. Todos somos iguais, mas temos de ter espeito às necessidades e as diferenças de cada um.

\subsection{Dificuldades no processo de inclusão}

$\mathrm{Na}$ integração escolar, o aluno tem acesso às escolas por meio de várias possibilidades educacionais, que abrange desde o acesso em ensino de escola especial até o ensino em salas de aula do Ensino Regular, dentro de uma concepção de inserção incompleta. Uma das dificuldades que chama atenção é que as escolas isolam os alunos com necessidades especiais e só integra os que não constituem nenhum tipo de desafio, isto ocorre quando se indica escolas de rede regular os que foram avaliados por instrumentos e profissionais específicos. Inserir aluno especial em uma sala de aula de ensino regular não faz dela inclusiva. (Ferraz \& Araújo; 2010).

Diante disso, o aluno com necessidades especiais só poderá estar em uma sala inclusiva quando a mesma atender, com qualidade, às necessidades educacionais para receber estes alunos, e poder dar um ensino de qualidade. A inclusão dar condições e possibilidades para que as pessoas com necessidades educacionais especiais possam realmente ter diretos igualados a todos e de usufruir da comunidade, com suas singularidades respeitadas. (Carreiro, 2010). 
Figura 2 - Importância da Inclusão.

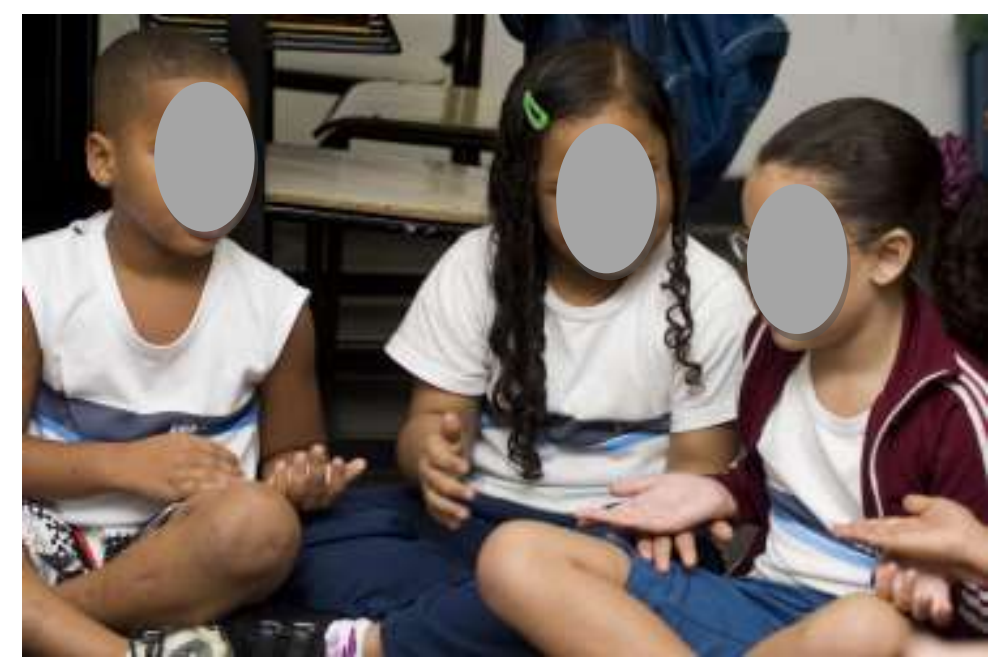

Fonte:http://www.movimentodown.org.br/2018/09/materiais-para-educacao/

Dentro da escola inclusiva, é imprescindível que crianças aprendam juntas, independentemente das diferenças, interagindo com todos, gerando sentimentos de respeito, comunicação, confiança, entre outras. Para que suprir as necessidades de seus alunos, a escola deve se organizar conforme os ritmos de aprendizagem, través dos currículos, alterações organizacionais e utilizar estratégica de ensino diferenciada (Mantoan, 2004)

De acordo com os autores, a educação inclusiva propõe a organização de escolas para que as mesmas sejam privilegiadas a e poder oferecer qualidade do atendimento especializado juntamente com o ensino da rede regular. Dessa forma, poder vivenciar a experiência de unir o aluno normal, com o aluno especial na verdadeira comunidade educacional, em que exista a aceitação, a solidariedade, a diversidade, o respeito e trabalho. Este é o objetivo principal da escola inclusiva aprender a trabalhar com as diferenças de ritmos de aprendizagem

\section{Aspectos da Inclusão Escolar: as Adaptações Curriculares e a Avaliação da Aprendizagem}

De acordo com a Constituição a educação inclusiva é um paradigma educacional fundamentado na concepção de direitos humanos, relacionando igualdade e diferença como valores indissociáveis. Podendo ser vista ação de cunho político, cultural, social e pedagógico em defesa dos direitos de todos os alunos estudarem juntos sem quaisquer tipos de discriminação e participação e aprendizagem (Brasil, 2008).

A proposta da inclusão escolar é oferecer um ensino de qualidade para todos com uma reestruturação do sistema escolar. Nessa perspectiva, as modificações que envolvem as alterações necessárias na política educacional para que seja eficaz; o projeto pedagógico; uma flexibilidade organizacional. Como também, provisão de recursos financeiros; acessibilidade a todos os ambientes físicos; parceria com os pais; gestão mais democrática; aproveitamento máximo dos recursos materiais e humanos; identificação e avaliação das necessidades educativas especiais. (Marchesi, 2004; Sousa, 2015).

De acordo com a constituição e os autores a inclusão escolar é um desafio constante no qual se questionam valores, crenças, concepções, normas, resistências, atitudes, com adaptações curriculares, novos métodos, técnicas, agrupamentos e formas de avaliação; formação continuada de professores, tudo em prol da união de um ensino que supre as necessidades de pessoas normas e especiais e que também, sejam vistas com igualdades por todos os envolvidos no processo e pela sociedade. 


\subsection{Avaliação da aprendizagem e o aluno com SD}

Segundo Luckesi (2008), afirma que a avaliação da aprendizagem deve servir de suporte no processo de adoção de atitudes metodológicas mais adequadas que busquem alcançar os objetivos, representando um meio que fortaleça o crescimento do educando. Pois, avaliação e planejamento estão articulados, representando um mecanismo subsidiário de planejamento e execução. (Oliveira \& Campos, 2005).

A avaliação é um processo complexo, pois, cada aluno tem sua individualidade em decorrência de sua deficiência. A avaliação processual e intencional de alunos com deficiência intelectual, para demonstrar o seu entendimento, mostrando o seu potencial e possibilidades de práticas favorecedoras de aprendizagem (Oliveira \& Pletsch, 2014; Valentim, 2011).

Hoffmann $(2009,2001)$ diz que o processo avaliativo está centrado nos aspectos educacionais, e não apenas no entendimento imediato; questiona a ideia de terminalidade, de finalização e de atribuição de notas ou conceitos e cálculo de médias no processo avaliativo. Para a autora, esse processo deve ser visto como mediações que incentivem o saber e a reorganização do mesmo, propiciando ações, movimentos e provocações que causam a troca do intelectual, de conhecimentos entre professores e alunos de forma reflexiva.

Conforme os autores as intervenções de avaliação adequada às especificidades dos aprendizes com SD, permitem estimular suas potencialidades, seus conhecimentos adquiridos. Os estudantes estão em evolução, em diferentes ritmos, e o professor deve estimulá-los a progredir sempre nessa perspectiva, o aluno com SD tem capacidade, assim como os demais alunos, de participar do processo de avaliação.

\section{Resultados Esperados}

Segundo Martins (2011, p.27 “[...] É considerada a patologia mais frequente, associada à deficiência intelectual, sendo responsável por cerca de $18 \%$ a $20 \%$ dos casos de déficits intelectuais existentes". A Síndrome de Down está agregada a deficiência intelectual, por haver excesso de material genético do cromossomo a mais no par 21, assim trazendo para a criança limitações no intelectual. Para o autor, "A deficiência intelectual não se constitui em um grupo homogêneo, mas em um complexo conjunto de síndromes [...], decorrentes de fatores pré-natais (genéticos e congênitos), perinatais e pós-natais". Afirmando que os fatores pré - natais são aqueles acontecem em todo o processo de concepção. As crianças com Síndrome de Down apresentam limitações, são lentas e demoram muito tempo para atingir a maturidade e desenvolver-se na linguagem e no aprendizado do que outras pessoas. Porém, elas podem desenvolver outras habilidades dentro de suas limitações.

Espera-se que através dessa pesquisa seja possível afirmar que é possível realizar um trabalho de inserção de crianças com deficiências e principalmente SD, pois são síndromes que surgem precocessimente e que prejudicam no processo de socialização, as crianças com estes problemans tem dificuldade de relacionamento e na aprendizagem. Pois se faz necessário um trabalho pedagógico diferenciado para a inserção destas crianças na educação infantil.

O intuito deste trabalho é demonstrar que é possivel essa inserção, e podemos afirmar através da pesquisa realizada, onde os dados demonstram a possibilidade deste processo, conforme os autores pesquisados, e que venha servi de relevância para estudos posteriores sobre o tema abordado.

\section{Considerações Finais}

O tema pesquisado buscou expressar como vem acontecendo e se vem acontecendo à inclusão de crianças com Síndrome de Down na educação de ensino regular, bem como as concepções de inclusão, as metodologias e as dificuldades que ocorrem no dia-a-dia dos professores e alunos durante este processo de inclusão. Tendo em vista isso, tornou-se curioso e interessante, tendo em vista que existe pouca literatura a respeito do tema abordado. 
Levando em conta o mundo atual, com os novos avanços da tecnologia presentes na sociedade atual, é necessário conscientizar e analisar as dificuldades da equipe de profissionais e principalmente os professores que encontram devido as diferenças, entende-se que não é somente a escola que deve submeter o papel da inclusão, e sim, toda a sociedade que ainda infelizmente tem muitos preconceitos, é indispensável ter um olhar mais humano diante das diferenças.

Dessa forma entendemos que o papel da escola e família é muito importante na conscientização das crianças perante as diferenças de raças, crenças, deficiências etc. Buscamos através desse trabalho, proporcionar uma melhor compreensão da inclusão social. Pois, a Síndrome de Down (SD) é entendida como uma alteração genética caracterizada pela presença de um cromossomo 21 a mais em todas as células do organismo do sujeito, levando-o a manifestar várias características físicas e mentais específicas que podem sem observadas em sua aparência desde do nascimento, porém, não se relacionam ao seu padrão de comportamento e desenvolvimento.

Em suma acredita-se que nas mudanças de atitudes dos profissionais de educação, da sociedade, das crianças e famílias perante aos portadores de Síndrome de Down, e com essa mudança de atitude pode-se realizar uma construção de uma sociedade diferente sem preconceitos, e assim contribuir na melhoria como cidadãos. Exaltando o tema com relação às dificuldades d inclusão no ensino percebe-se que existe problemas e desafios para que a inclusão ocorra de fato, dentre outros aspectos podemos destacar: falta de recursos como livros, brinquedos educativos e por outro lado, salas superlotadas, faltas excessivas de alunos, baixos salários, falta de acompanhante para alunos especiais, entre outros. Como a aplicação de novas alternativas como também novas práticas pedagógicas educacionais compatíveis com a inclusão, que podem prejudicar o ensino inclusivo.

\section{Referências}

Aranha, M. S. F. (2001). Inclusão social e municipalização. In: Novas Diretrizes da Educação Especial. São Paulo: Secretaria Estadual de Educação.“

Bardin, L. (2011). Análise de conteúdo. 70.

Blanco, R. (2004).A atenção à diversidade na sala de aula e as adaptações do currículo. In: Coll, C., Marchesi, 290-308.

Brasil. (1988). Constituição de 1988. Constituição da República Federativa do Brasil. Diário Oficial [da] República Federativa do Brasil, Poder Executivo, Brasília, DF

Brasil. (1997). Declaração de Salamanca e linha de ação sobre necessidades educativas especiais. (2a ed.), Corde.

Brasil. (2008). Ministério da Educação. Secretaria de Educação Especial. Política Nacional de Educação Especial na perspectiva da Educação Inclusiva. Brasília, DF: MEC.“.

Brasil. (2008). Ministério da Educação. Secretaria de Educação Especial. Resolução CNE/CEB n. 4, de 2 de outubro de 2009. ed. VOZES“.

Castro, A., \& Pimentel, S. Atendimento educacional específico. Síndrome de Down: desafios e perspectivas na inclusão escolar. In: Díaz, F. et al. (Org.). Educação inclusiva, deficiência e contexto social: questões contemporâneas. UFBA, 2009. p. 303-312.

Ferraz, C., Araújo, M., \& Carreiro, L. (2010).Inclusão de crianças com Síndrome de Down e paralisia cerebral no ensino fundamental: comparação dos relatos de mães e professores. Revista Brasileira de Educação Especial, 16(3), 397-414.

Mantoan, M. T. E. (2011).Inclusão escolar: O que é? Por quê? Como fazer? São Paulo: Moderna, 2003. Martins, L. de A. R. Fundamentos em educação inclusiva. Editora UFRN.

Mantoan, M. T. E. (2004).Inclusão escolar: o que é? Por quê? Como fazer? Moderna, 2004.

Santos Filho, J. C. dos. (2001). Pesquisa quantitativa versus pesquisa qualitativa: o desafio paradigmático. In: Santos Filho, J. Camilo dos, Gamboa, Silvio Sánchez. Pesquisa educacional: quantidade-qualidade. (4a ed.), Cortez, p.13-59.

Stratford, B. (1997).Crescendo com a Síndrome de Down. Tradução: Lucia Helena Reilly, Revisão: Viviane Veras, Revisão técnica: A. Fernando Ribeiro, Ulysses Moraes de Oliveira. Corde.

Schwartzan, J. S. (1999).Síndrome de Down. Mackenzie.

Tartuce. T. (2019).Normas Técnicas para trabalhos acadêmicos/ Teresinha Tartuce. - Fortaleza: Expressão Gráfica e Editora, p. 65.

Pueschel, S. M. (1993).Síndrome de Down: guia para pais e educadores. Papirus. 
Research, Society and Development, v. 10, n. 16, e317101622702, 2021

(CC BY 4.0) | ISSN 2525-3409 | DOI: http://dx.doi.org/10.33448/rsd-v10i16.22702

Voivodic, M. A. Inclusão escolar de crianças com síndrome de Down. 5.

Voivodic, M. A. M. A. (2013).Inclusão escolar de crianças com Síndrome de Down. (7a ed.), Vozes

\section{Revista Consultada}

Educação \& Formação Revista do Programa de Pós-Graduação em Educação da Universidade Estadual do Ceará (UECE) Educação \& Formação, 3(9), 121140, set./dez. 2018.

\section{Sites consultados}

http://cerbrum.com/inclusao-escolar-promove-justica/

http://www.movimentodown.org.br/2018/09/materiais-para-educacao/ 